\title{
A breakthrough instrument for wide- range wavelength and angle resolved light scattering measurements of high performances optical coatings
}

Marin Fouchier, Myriam Zerrad, Michel Lequime, Claude Amra

Marin Fouchier, Myriam Zerrad, Michel Lequime, Claude Amra, "A breakthrough instrument for wide-range wavelength and angle resolved light scattering measurements of high performances optical coatings," Proc. SPIE 11852, International Conference on Space Optics — ICSO 2020, 118521C (11 June 2021); doi: 10.1117/12.2599236

SPIE Event: International Conference on Space Optics - ICSO 2021, 2021, Online Only 


\section{International Conference on Space Optics-ICSO 2020}

Virtual Conference

30 March-2 April 2021

Edited by Bruno Cugny, Zoran Sodnik, and Nikos Karafolas
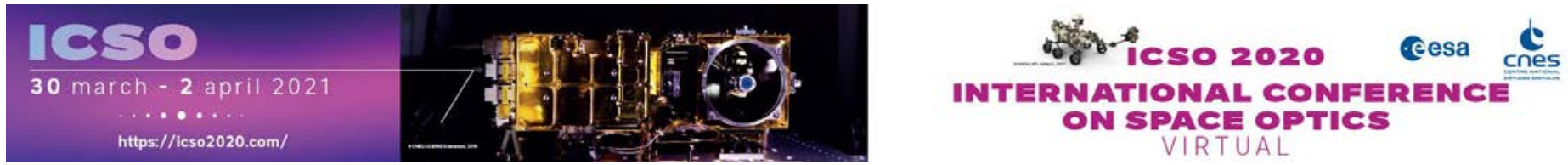

\section{A breakthrough instrument for wide-range wavelength and angle resolved light scattering measurements of high performances optical coatings}

\section{Cesa issopocestings lenes}




\title{
A breakthrough instrument for wide-range wavelength and angle resolved light scattering measurements of high performances optical coatings
}

\author{
Marin Fouchier*ab, Myriam Zerrad ${ }^{\mathrm{a}}$, Michel Lequime ${ }^{\mathrm{a}}$, Claude Amra ${ }^{\mathrm{a}}$ \\ ${ }^{a}$ Aix Marseille Univ, CNRS, Centrale Marseille, Institut Fresnel, Marseille, France \\ ${ }^{\mathrm{b}}$ Centre National d'Études Spatiales, France \\ *marin.fouchier@fresnel.fr
}

\begin{abstract}
Due to the diversity and complexity of optical functions they address, optical coatings are widely used in space optics but the level of both their specifications and performances is now so high that the characterization of their optical properties requires the development of extreme metrology. In this context we present in this paper the last state-of-the-art instrument for thin-films characterization. This apparatus is unique by the performances and the levels of detectivity achieved. It operates spectrally and angularly resolved characterization of Angle Resolved Scattering (ARS) over a wide and continuous spectral range from $400 \mathrm{~nm}$ to $1.7 \mu \mathrm{m}$. The spectral resolution is lower than $1 \mathrm{~nm}$, while the angular resolution is lower than $1^{\circ}$ with a precision better than $1 \%$. The lowest levels of scattering measured are close to $10^{-8} \mathrm{sr}^{-1}$, near to the limit of air particles scattering. The large angular range available offers the opportunity to evaluate scattering functions both in the forward and backward half-spaces. In specific configurations, this same setup records specular properties, achieving again unreached levels of detection and precision over the same spectral range and with the same resolution. All measurements have shown great agreement with theoretical designs and numerical predictions, for both scattering and transmittance records. This instrument is an upgraded version of an apparatus developed in the Scattering group for more than five years formerly operating in the visible wavelength spectrum and now extended to near-infrared with the same level of performances.
\end{abstract}

Keywords: light scattering, optical densities, metrology, optical thin-films

\section{INTRODUCTION}

Optical thin-film components are widely used for space applications from Earth imaging [1] to green house gas monitoring [2] and gravitational waves detection [3]. The levels of both their specifications and performances are so high that they require very strong and accurate metrology. Light scattering phenomena severely affect components performances by reducing or distorting light signal or altering image quality. Accurate and performant metrology tools are needed to qualify these space dedicated components. SALSA (Spectral and Angular Light Scattering Apparatus) is a spectrophotometric instrument, spectrally and angularly resolved, which performs such a characterization. The instrument emerges from a more than five years development process. Previous publications have presented its operation in the visible range (400 $1000 \mathrm{~nm}$ ) for both scattered light and specular functions estimation [4]-[7]. This paper presents an upgraded version of the SALSA instrument carrying out measurements in the visible and the near-infrared spectra, from 400 to $1700 \mathrm{~nm}$. Its detection limit is closed to the air particle scattering level $\left(\sim 10^{-8} s r^{-1}\right)$ while it measures up to 13 optical densities (ODs) on specular functions.

Many instruments have been proposed for this metrology but most of them use laser lines to reach sufficient power and measure scattering at discrete wavelengths in the UV, visible or infrared [8], [9]. The configuration of the setup presented in this paper overcomes this limitation and owns a continuous spectral range. 


\section{PRESENTATION OF SALSA INSTRUMENT}

\subsection{Design of the instrument}

SALSA sits in an ISO6 clean room facility that preserves it from general environmental variations and dusts. Figure 1 shows a functional representation of the SALSA instrument composed of different subassemblies. The emission subassembly is based on a supercontinuum laser source (FIANIUM WL-SC-400-8) which outputs a very wide spectrum light signal from $400 \mathrm{~nm}$ to $2.4 \mu \mathrm{m}$ with a total integrated power of $8 \mathrm{~W}$. A dichroic beam splitter (FIANIUM Splitter950-01) splits the broadband spectrum into two channels: a visible channel (from 400 to $975 \mathrm{~nm}$ ) and an infrared channel (from $975 \mathrm{~nm}$ to $2.4 \mu \mathrm{m}$ ).

The optical components used on both channels are functionally identical but differ on their spectral operation ranges. The two channels are used independently and asynchronously, if one is activated then the other one stay closed. Two tunable volume hologram filters (Photon Etc. LLTF Contrast VIS HP8 and LLTF Contrast SWIR HP8 respectively) set the working wavelength and reduce the spectral width of the signal between 2 and $3.5 \mathrm{~nm}$. Electro-mechanical shutters (SH1 and $S H 2)$ block the light beam and transmit it only during acquisitions. Order sorting filters (OSF1 and OSF2) clean the signal from parasitic harmonic orders induced by the volume hologram filters. Mirrors $(M 1, M 2$ and $M 3)$ spatially align the optical beams and beam splitters ( $B S 1$ and $B S 2$ ) collect a small portion of the signal, around $10 \%$, as reference signals. Then the main signal goes through a set of four neutral optical density stages $(O D s)$. The combination of these densities results in a total OD between 0 and 10 , adjustable by a pitch of 1 . The only structural difference between the visible channel and the infrared channel is a removable recombination mirror $(R M)$ which reflects the visible channel towards the optical densities. This mirror is mounted on a translation stage, when performing acquisitions in the visible spectrum the mirror reflects the light beam towards the output of the emission subassembly, while it clears the way for the infrared channel when performing infrared acquisitions. The light beam is finally collected by a parabolic off-axis reflective collimator (ORC). The reference signals collected by the above-mentioned beam splitters ( $B S 1$ and $B S 2)$ are also sent to reflective collimators ( $R C R I$ and $R C R 2$ ) connected to photodiodes (VIS_PD and $I R \_P D$, respectively FEMTO DE-OE-200-SI and DE-OE200-In1) via multimode optical fibers (FOL $R 1$ and $\bar{F} O L R 2)$.

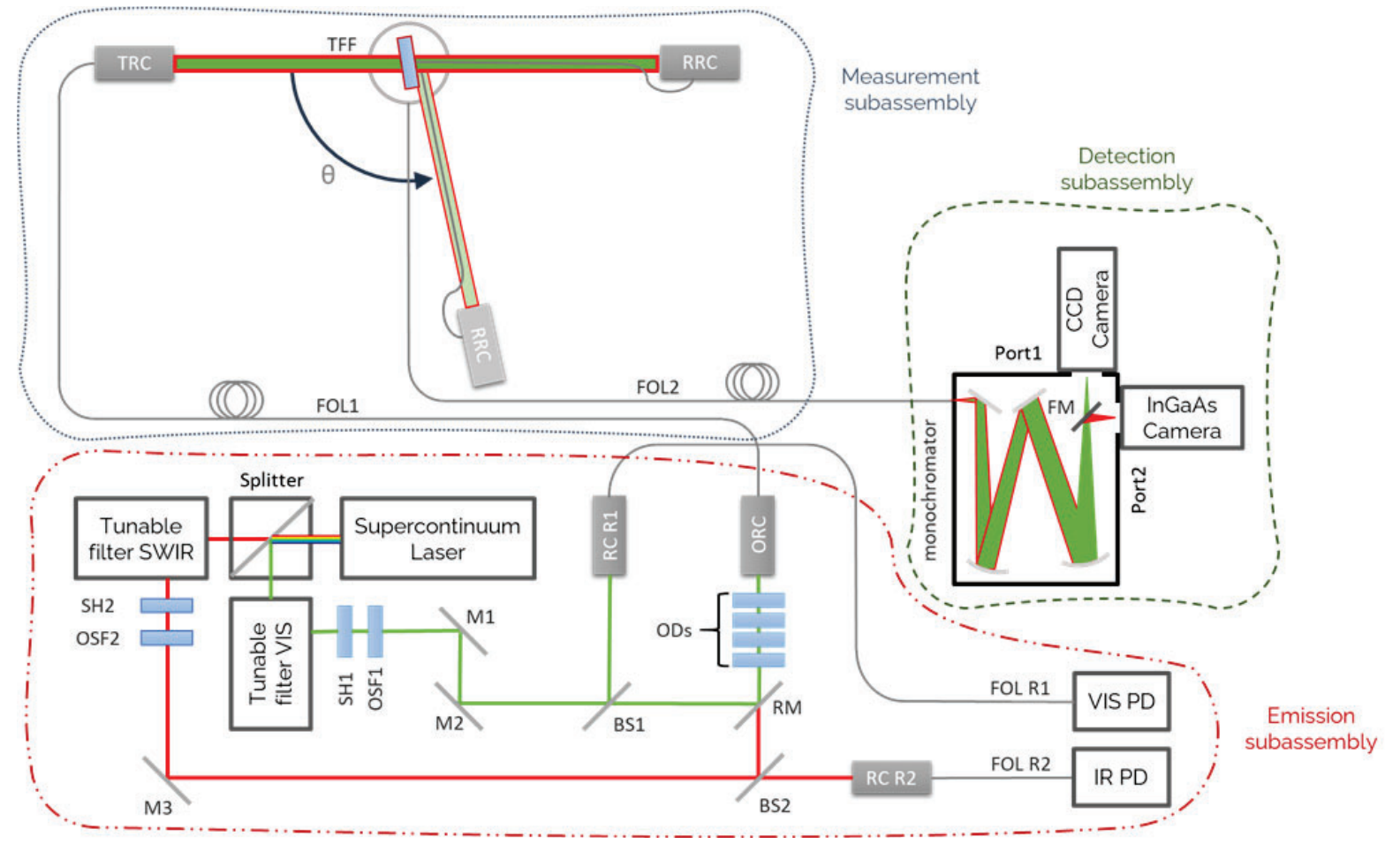

Figure 1 Spectral and Angular Light Scattering Apparatus (SALSA) is composed of three subassemblies: Emission, Measurement and Detection. 
The measurement subassembly is connected to the emission subassembly with a multimode fiber optical link (FOL1, core diameter $50 \mu \mathrm{m})$. A reflective collimator (TRC) outputs the optical beam which illuminates the sample under characterization $(T F F)$. The incidence angle is set by a rotation stage that holds the sample. After being transmitted, reflected, or scattered, light is collected by a second reflective collimator $(R R C)$ mounted on a rotation arm centered on the sample. Therefore, by adjusting the angular position $\theta$ of the mechanical arm, it is possible to measure scattered light at any angle around the sample and in the plane of incidence. The measurement subassembly is connected to the detection subassembly with a multimode fiber optical link (FOL2, core diameter $105 \mu \mathrm{m})$.

The detection subassembly includes a Czerny-Turner monochromator (ANDOR Shamrock SR-193i-B1-SIL), two gratings are mounted back-to-back on a turret in the spectrograph. The first one is an 1800-lines-per-milimeters holographic grating optimized for a range from 360 to $1100 \mathrm{~nm}$. The second one is a 760-lines-per-milimeters optimized for $850 \mathrm{~nm}$ to $2 \mu \mathrm{m}$. The spectrograph owns two output ports, on each is mounted a scientific grade camera: a CCD detector on port1 (ANDOR Newton DU920P-BVF) with $255 \times 1024$ pixels of $26 \times 26 \mu \mathrm{m}$ each and an InGaAs linear detector on port2 (ANDOR DU491A) with $1 \times 1024$ pixels of $500 \times 25 \mu \mathrm{m}$ each. A sliver-coated flip mirror $(F M)$ leads the light beam to one of the two ports. The CCD camera detects visible light, while InGaAs is sensitive to the infrared spectrum.

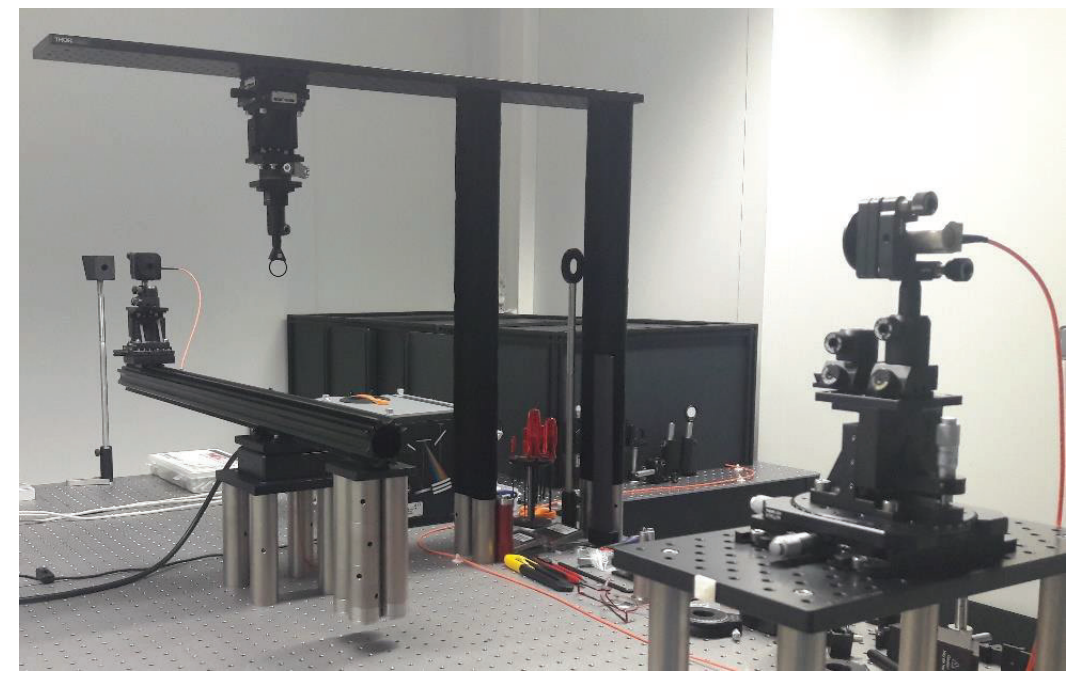

Figure 2 SALSA instrument in ISO6 clean room in the Espace Photonique of Institut Fresnel. Measurement subassembly: a reflective collimator (right) outputs the optical beam which illuminates the sample (middle), the scattered light is collected by a second reflective collimator mounted on a rotating arm (left).

\subsection{Operation of the instrument}

The measurement procedure has been already presented in our previous papers [5]-[7]. For measuring the amount of scattered light by any sample at a wavelength $\lambda_{0}$ and an angle $\theta_{0}$, the rotating arm is set at the chosen angle. The value of $\lambda_{0}$ determines which channel (visible or infrared) to use in the emission and detection subassemblies. The involved LLTF filter, the recombination mirror, the spectrometer grating, and the detection camera are tuned to the chosen $\lambda_{0}$ value. Initial values are set for the $\tau$ (exposition time) and $O D$ (overall optical densities) parameters. Then the electromechanical shutter is opened to let the light beam illuminate the sample. The acquisitions on the reference photodiode and the camera are synchronized thanks to this single shutter. The signal on the camera is expected to be around $70 \%$ of its saturation level. The effective level is adjusted by tuning the $\tau$ and $O D$ parameters simultaneously. Several acquisitions might be executed until the signal achieves a convenient level. The CDD camera has numerous lines of pixels and the light beam illuminates only a small area on the detector. The illuminated area is small enough to acquire simultaneously the signal and the noise using different zones of the detector matrix. On the other hand, the InGaAs camera has only one line of pixels which collect the signal. It is not possible to acquire the signal and the noise simultaneously. The noise level is measured immediately after the signal with another acquisition of the same duration but with no illumination of the sample. Depending on the position of the rotating arm, SALSA measures either the scattered light or the specular transmitted or reflected light. The scattering function estimated is the Angle Resolved Scattering (ARS), a spectrally and angularly resolved function which describes the angular distribution of the scattered light around the sample. The specular functions estimated are the 
transmittance and the reflectance. In any case a calibration is required. A Spectralon white diffuse reflectance sample defines the standard levels for the scattering measurements, while a baseline (with no sample) acquisition defines the reference level for specular functions measurements.

\section{SAMPLE CHARACTERIZATION AND COMPARISON TO MODELS}

\subsection{Light scattering characterization}

A dedicated bandpass filter centered at $1273 \mathrm{~nm}$ has been used for the qualification of the instrument, this sample is called F0 in this paper. This filter has been designed and manufactured by CNES and CILAS ArianeGroup and is intended for a space application. It is composed of around 100 alternating layers of $\mathrm{SiO}_{2}$ and $\mathrm{Nb}_{2} \mathrm{O}_{5}$. The calibration of the setup requires the measurement of the ARS of a perfect white reflectance Lambertian diffuser (LABSPHERE Spectralon). The ARS analytical formulation for this material is the following:

$$
\operatorname{ARS}(\lambda, \theta)=\frac{1}{\pi} \rho(\lambda) \cos \theta
$$

Where $\rho(\lambda)$ is the albedo of the sample at wavelength $\lambda$, the angular cosine dependance is a specific feature used to check the correct alignment of the setup. The ARS measured on this sample is compared to a cosine shape, a good agreement assesses a right optical alignment of the sample and the rotating arm.

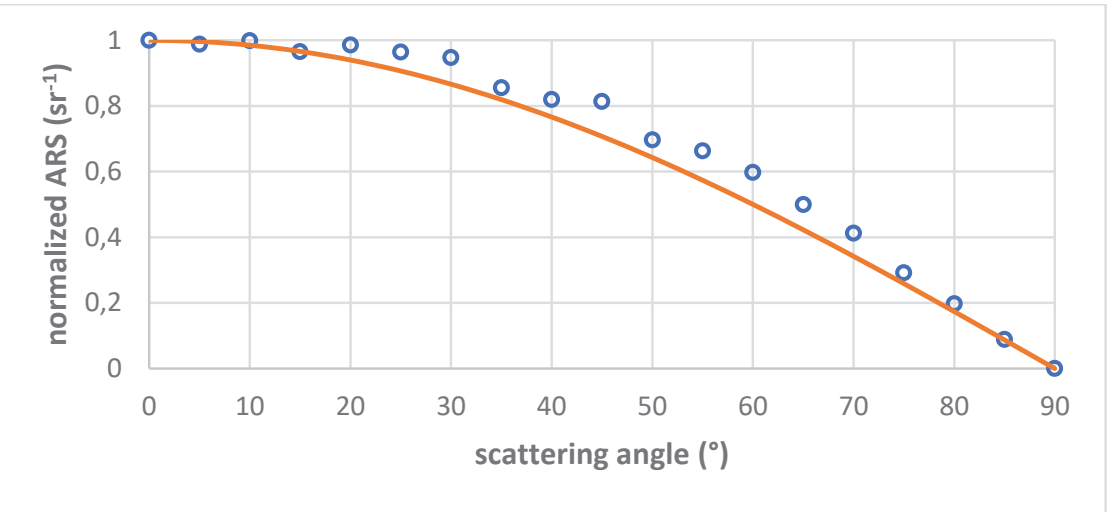

Figure 3 Comparison between the ARS cosine model and the normalized ARS measured on the Spectralon sample at $1490 \mathrm{~nm}$.

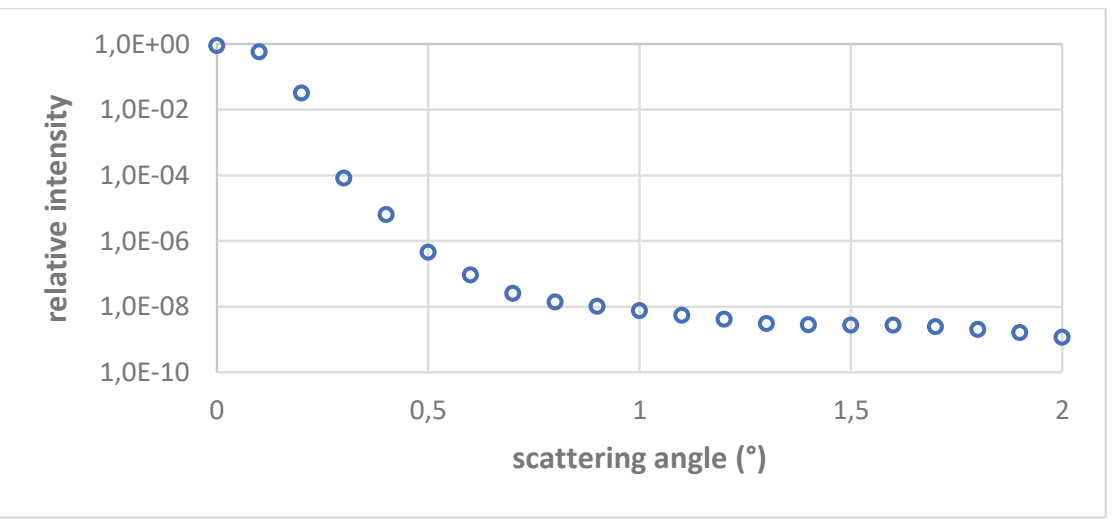

Figure 4 Angular response of the instrument to the direction defined by the transmitted specular beam.

The angular resolution is granted by the constitution of the setup and the use of a high-precision rotation stage. The angular response presents a relative decrease of 8 decades at $1^{\circ}$ from the specular beam position. Therefore, we can confidently assert that the angular resolution of the setup is below $1^{\circ}$. 
ARS measurements on the sample F0 show both spectral and angular variations. Recent works carried out in our group have led to a complete and powerful theoretical model implementation which describe such scattering from optical thinfilms [10]. The comparison between the model and the measurements presents an excellent and unprecedented agreement. Not to forget, the sample under characterization is very complex which makes it difficult to characterize experimentally and numerically. The model also highlights the spectral and angular peaks observed experimentally. An acquisition without sample depicts the Signature of the instrument, i.e., the lowest detectable values. The level of the Signature shows that the setup should be able to measure down to $10^{-8} s r^{-1}$, closed to the air particles scattering limit. F0 has been characterized at some specific wavelengths, Figure 5 and Figure 6 depict the measurement and model data at $1115 \mathrm{~nm}$ and $1270 \mathrm{~nm}$ with a fine angular resolution $(1 \mathrm{~nm})$. The same comparison has been carried along all the near-infrared accessible spectrum with a lower angular resolution and the agreement with the model remains excellent.

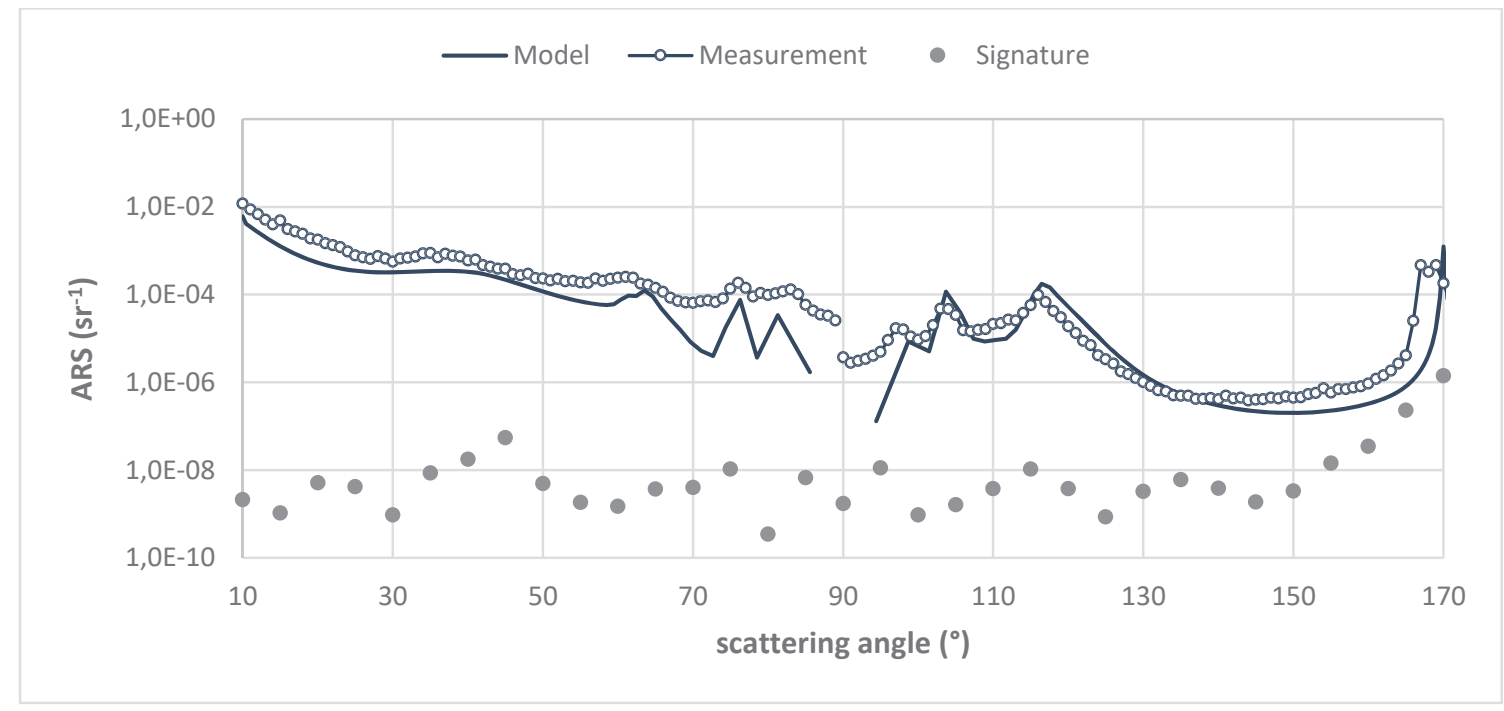

Figure 5 ARS measured on F0 at $1115 \mathrm{~nm}$. Comparison with Model and Signature.

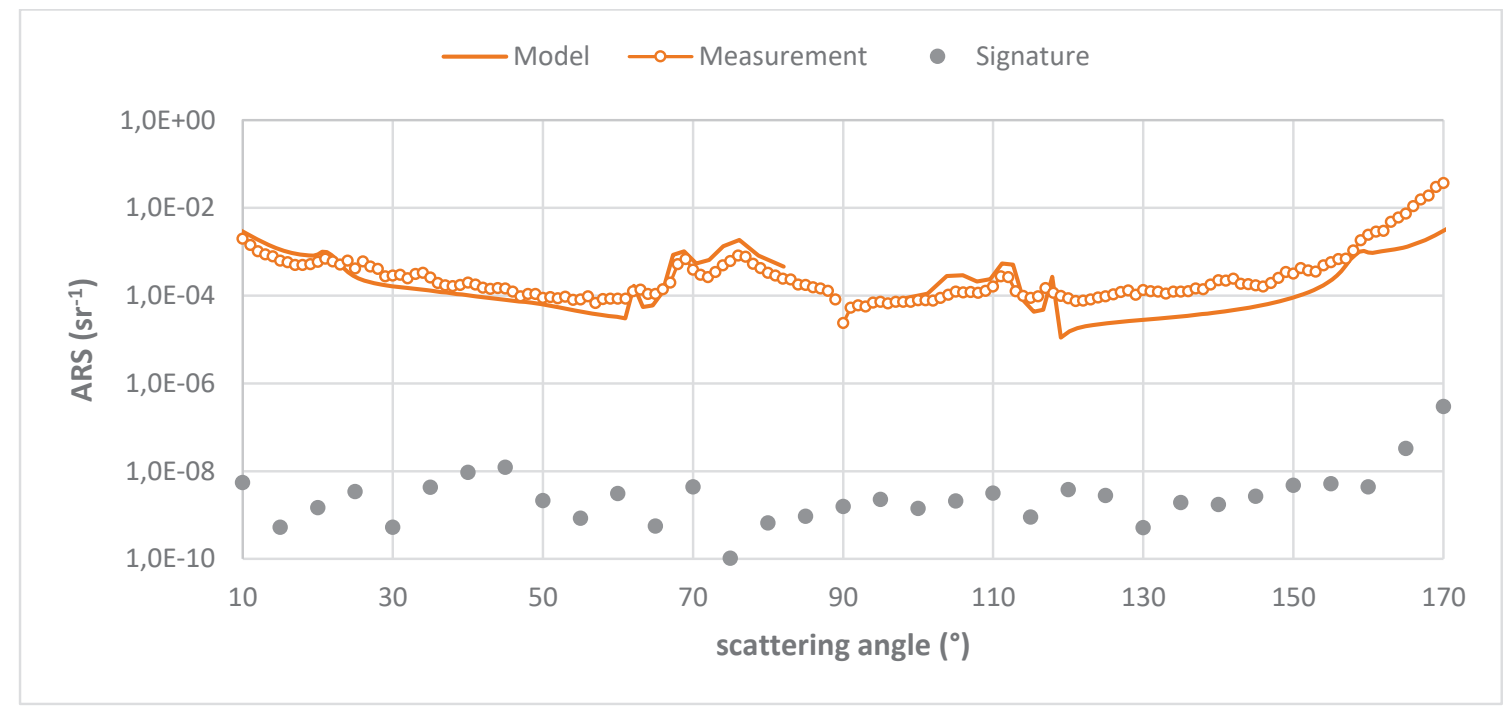

Figure 6 ARS measured on F0 at $1270 \mathrm{~nm}$. Comparison with Model and Signature. 


\subsection{Specular transmittance characterization}

The same F0 filter has been subjected to specular transmittance characterization, illustrated on Figure 7 . The measurement data shows an excellent agreement with the design data: the high rejection levels are well defined, as well as the narrow spectral peaks and the steep edges. These results validate the asserted that the spectral resolution is lower than $1 \mathrm{~nm}$. Once again the Signature of the instrument is very low, around $10^{-12}$, which is 6 decades below the state-of-the-art [11].

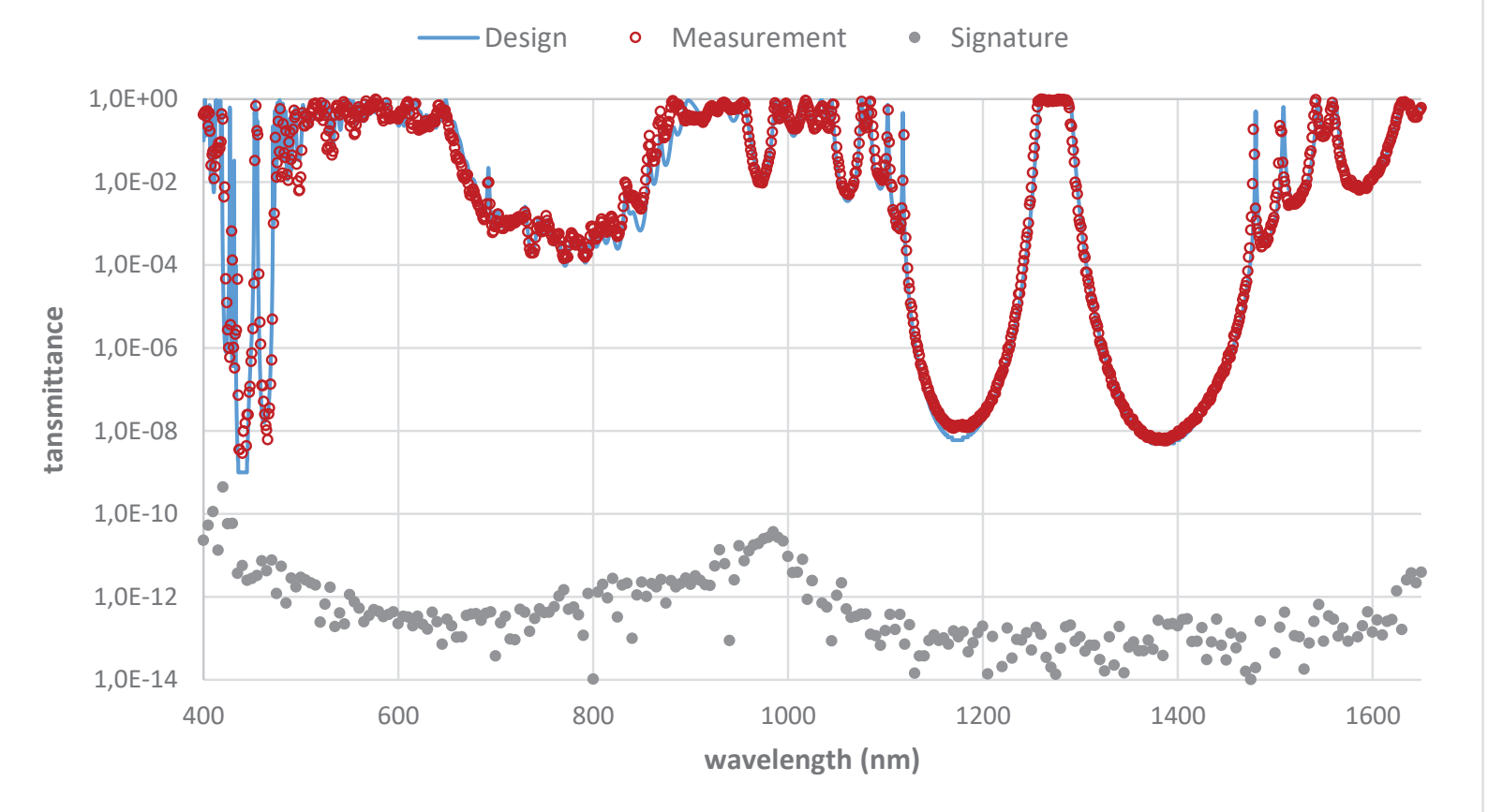

Figure 7 Transmittance of the dedicated F0 filter from 400 to $1650 \mathrm{~nm}$. Comparison between the design, the measurement, and the Signature of the instrument.

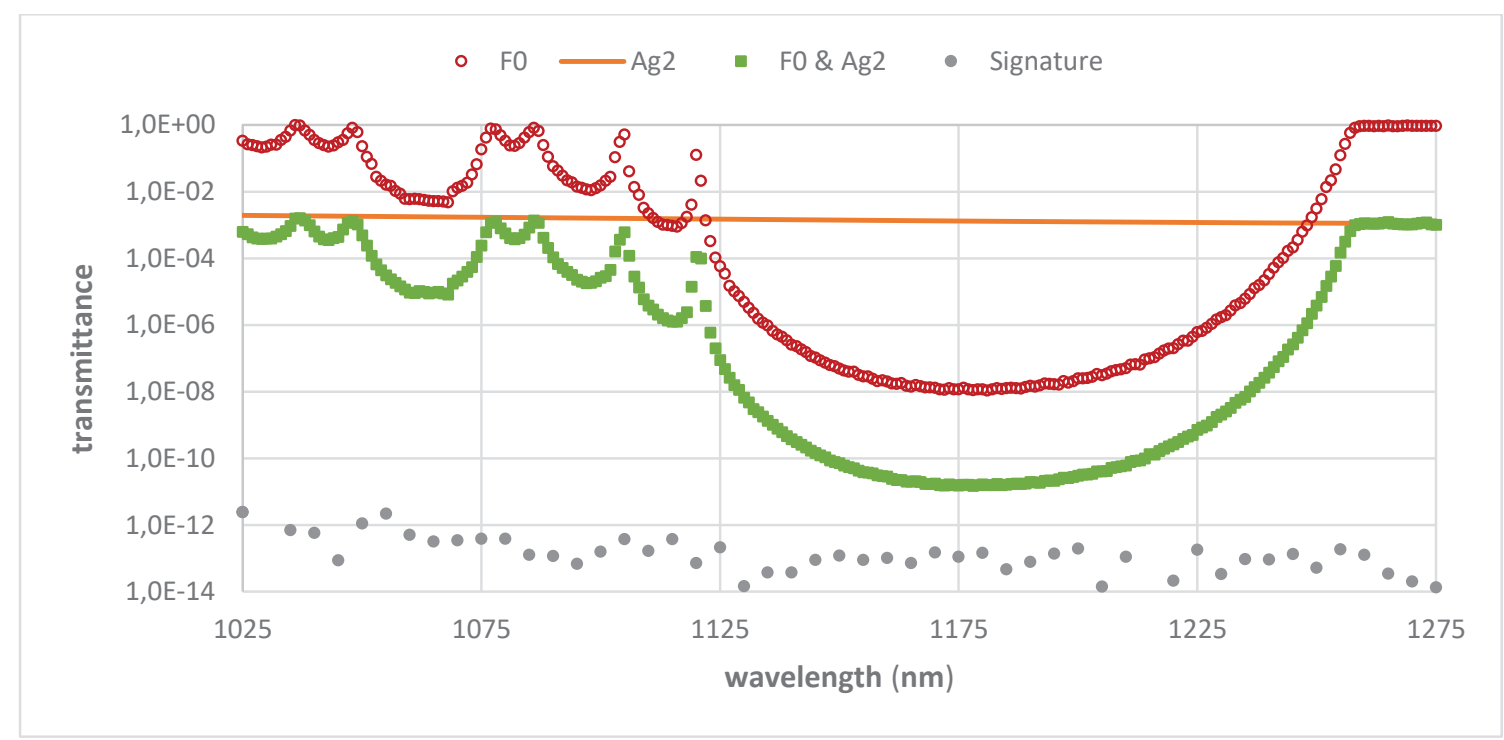

Figure 8 Transmittance of F0 superposed with a silver monolayer density (Ag2) compared with single F0 and single Ag2 on the [1025-1275 nm] band. The resulting transmittance is close to the Signature level. 
The rejection of the above-mentioned optical filter is considerably larger than the noise level. To validate the detection limits, a $60 \mathrm{~nm}$ thick silver monolayer neutral density, called Ag2, is superimposed on F0. This optical density has a transmittance around $10^{-3}$ in the near infrared range. The total transmittance of the two components superposition is measured between $1025 \mathrm{~nm}$ and $1275 \mathrm{~nm}$, roughly centered on one of the two main deep rejection bands. The resulting transmittance value is the one of the F0 filter lowered by a factor of $10^{-3}$. The lowest level achieved in this configuration is $10^{-11}$, close to the Signature limit.

\subsection{Improve the spectral resolution}

The measurement campaign for F0 characterization over the whole available spectrum counts 1301 data points, from $400 \mathrm{~nm}$ to $1.7 \mu \mathrm{m}$ by step of $1 \mathrm{~nm}$. The resulting resolution is $1 \mathrm{~nm}$, but it can be improved with an adequate data postprocessing. The combination of a monochromator with an array-based detector shows a great interest in terms of spectral discrimination. The light beam arriving at the monochromator has a small spectral bandwidth (between 2 and $3.5 \mathrm{~nm}$ ) dispersed spatially by the grating. The signal acquired by the camera is spread over a horizontal line of pixels Considering the dispersion of the grating it is possible to discretize the wavelengths closed to the working one using different pixels of the camera. This configuration cleans the signal from non-desired spectral contributions and increases the spectral resolution. The instrument can consequently resolve narrow spectral peaks $(<1 \mathrm{~nm})$. Figure 9 illustrates this spectral dispersion on the raw signals detected by the cameras, the blue points represent baseline acquisitions (no sample), the red points represent acquisitions with sample. The presence of a sample under characterization modifies the signals and provides information about the spectral function locally. The spatial information along the pixel line is transcribed into spectral information. Figure 10 depicts a high-resolution processing, resulting in a transmittance resolution of $0.1 \mathrm{~nm}$ obtained from a $1 \mathrm{~nm}$ resolution acquisition. The comparison with the high-resolution processing is excellent and suggests that the narrow spectral peaks are even better resolved. The transmittance levels are displayed only between $10^{-4}$ and 1 on vertical axis and from $1075 \mathrm{~nm}$ to $1300 \mathrm{~nm}$ for ease of reading.
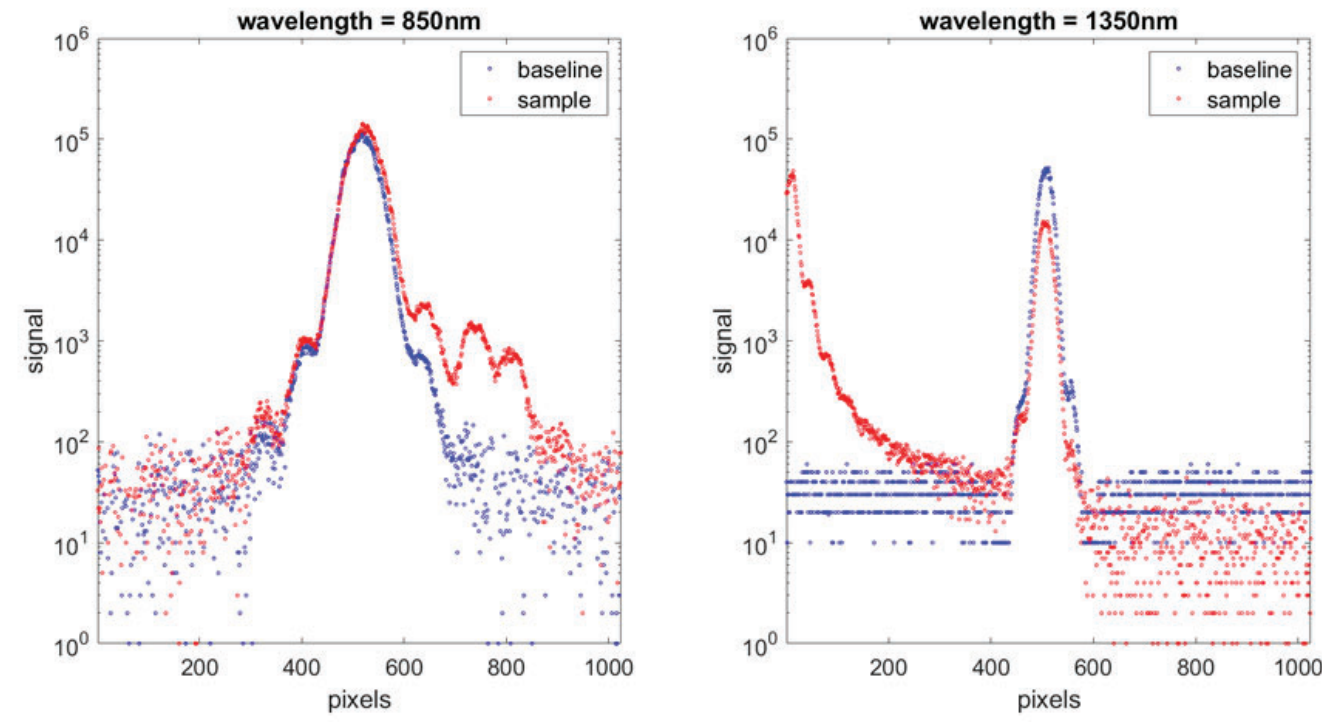

Figure 9 Raw signals acquired on the 1024 pixels line. The spatial position corresponds to a spectral information around the working wavelength (left $850 \mathrm{~nm}$, right $1350 \mathrm{~nm}$ ). 


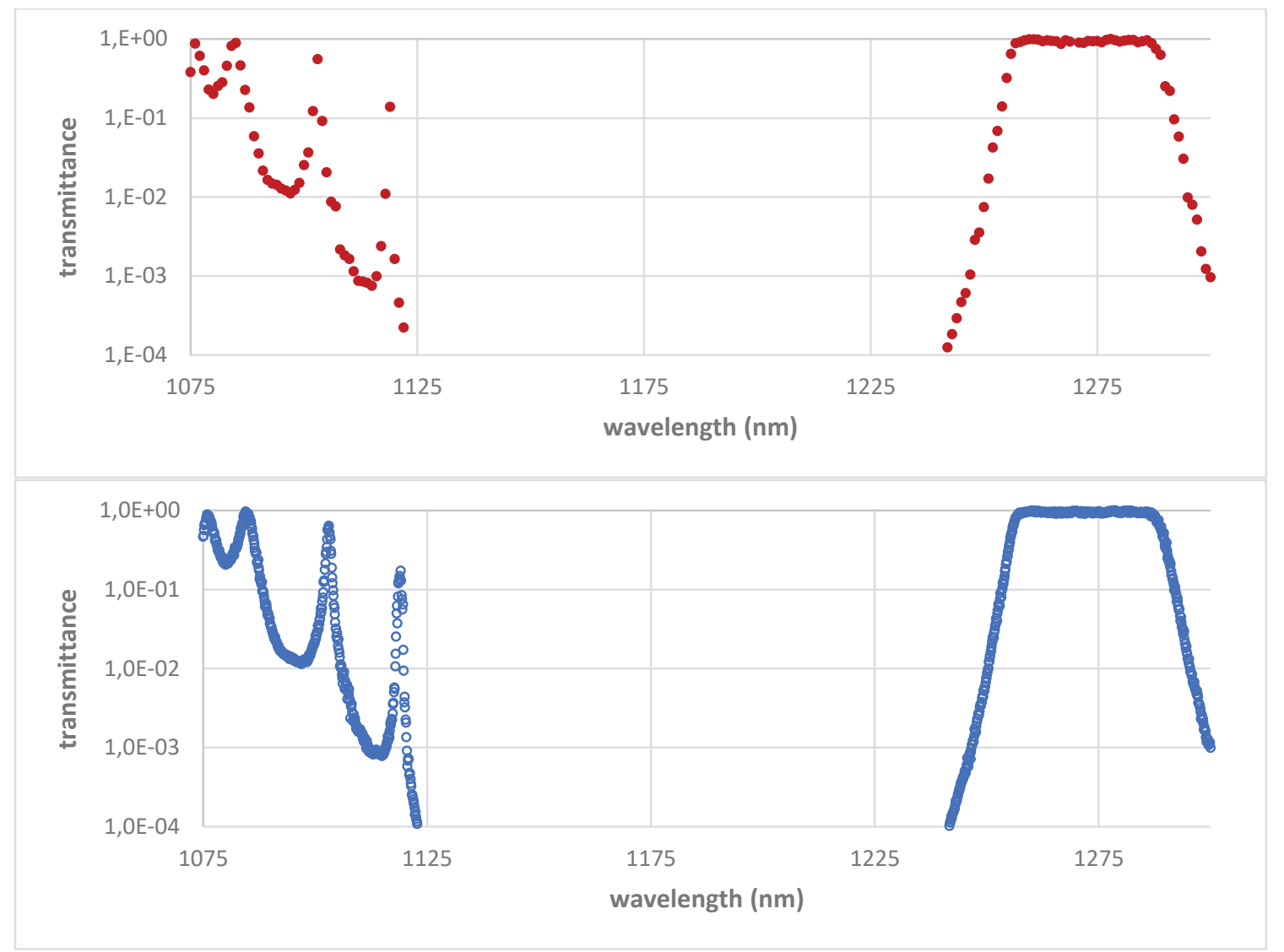

Figure 10 Top: transmittance of sample F0 with $1 \mathrm{~nm}$ spectral resolution. Bottom: transmittance of sample F0 with high-resolution processing. The high-resolution processing is coherent with the classical processing and improves the spectral information.

\section{CONCLUSION}

We have presented an angularly and spectrally resolved scatterometer that performs optical thin-film components characterization. The setup outperforms other existing instruments and offers very broad and continuous spectral and angular ranges. The reached detection limits are $10^{-8} \mathrm{sr}^{-1}$ for scattered light and $10^{-13}$ for transmittance measurements in the SWIR. This instrument developed over the last years is now operating in the visible as in the near-infrared and it is part of the DIFFUSIF metrological platform [12]. The performances have been qualified with the characterization of a dedicated filter intended for space applications.

\section{ACKNOWLEDGEMENT}

The authors would like to thank CNES (Centre National d'Etudes Spatiales), CILAS ArianeGroup, DGA (Direction Générale de l'Armement) and LabTOP (joint laboratory between CILAS ArianeGroup and Institut Fresnel) for their contribution to this work.

\section{REFERENCES}

[1] R. L. Goff et al., "Multispectral filters assemblies for earth remote sensing imagers," in International Conference on Space Optics - ICSO 2014, Nov. 2017, vol. 10563, p. 1056305, doi: 10.1117/12.2304107. 
[2] Frederick Pasternak, Philippe Bernard, Laurent Georges, and Véronique Pascal, "The microcarb instrument," Sep. 2017, vol. 10562, doi: 10.1117/12.2296225.

[3] H. Audley et al., "Laser Interferometer Space Antenna," Feb. 2017.

[4] S. Liukaityte, M. Lequime, M. Zerrad, T. Begou, and C. Amra, "Broadband spectral transmittance measurements of complex thin-film filters with optical densities of up to 12,” Opt. Lett., vol. 40, no. 14, pp. 3225-3228, Jul. 2015, doi: 10.1364/OL.40.003225.

[5] M. Lequime, M. Zerrad, and C. Amra, "Breakthrough spectrophotometric instrument for the ultra-fine characterization of the spectral transmittance of thin-film optical filters," Opt. Express, vol. 26, no. 26, p. 34236, Dec. 2018, doi: 10.1364/OE.26.034236.

[6] M. Lequime, S. Liukaityte, M. Zerrad, and C. Amra, "Ultra-wide-range measurements of thin-film filter optical density over the visible and near-infrared spectrum," Opt. Express, vol. 23, no. 20, p. 26863, Oct. 2015, doi: 10.1364/OE.23.026863.

[7] M. Fouchier, M. Zerrad, M. Lequime, and C. Amra, "Wide-range wavelength and angle resolved light scattering measurement setup," Opt. Lett., vol. 45, no. 9, pp. 2506-2509, May 2020, doi: 10.1364/OL.392000.

[8] A. von Finck et al., "International round-robin experiment for angle-resolved light scattering measurement," Appl. Opt., vol. 58, no. 24, pp. 6638-6654, Aug. 2019, doi: 10.1364/AO.58.006638.

[9] M. Hauptvogel et al., "Light scattering characterization of optical components for space applications," in International Conference on Space Optics - ICSO 2016, Sep. 2017, vol. 10562, p. 1056213, doi: 10.1117/12.2296113.

[10] C. Amra, M. Lequime, and M. Zerrad, Electromagnetic Optics of Thin-Film Coatings: Light Scattering, Giant Field Enhancement, and Planar Microcavities. Cambridge University Press, 2020.

[11] A. Johansen, A. Czajkowski, M. Scobey, P. Egerton, and R. Fortenberry, "THIN-FILM INTERFERENCE FILTERS FOR LIDAR," Alluxa White Pap., p. 12, Apr. 2017.

[12] "Diffusif." https://www.fresnel.fr/diffusif. 